\title{
Serum amyloid $A$ is a better predictive biomarker of mucosal healing than C- reactive protein in ulcerative colitis in clinical remission
}

\author{
Masaki Wakai', Ryohei Hayashi², Shinji Tanaka², Toshikatsu Naito', Junko Kumada', Motonobu Nomura',
} Hidehiko Takigawa', Shiro Oka', Yoshitaka Ueno ${ }^{2}$, Masanori Ito' and Kazuaki Chayama ${ }^{1}$

\begin{abstract}
Background: Many studies have revealed that mucosal healing improves the long-term prognosis of ulcerative colitis. Frequent colonoscopy is difficult because of its invasiveness and cost. Therefore, in diagnosing and treating ulcerative colitis, noninvasive, low-cost methods for predicting mucosal healing using useful biomarkers are required in the clinical setting.

This study aimed to evaluate whether serum amyloid $\mathrm{A}$ is a better serum biomarker than C-reactive protein in predicting mucosal healing in ulcerative colitis patients in clinical remission.
\end{abstract}

Methods: Ulcerative colitis patients whose C-reactive protein and serum amyloid A were measured within 1 month before and after colonoscopy were included in this retrospective study, and the relationship between the C-reactive protein and serum amyloid A values and the mucosal condition was analyzed. Mucosal condition was assessed using the Mayo Endoscopic Score, with score 0 or 1 indicating mucosal healing.

Results: A total of 199 colonoscopic examinations were conducted in 108 ulcerative colitis patients who underwent C-reactive protein and serum amyloid A blood tests. In clinical remission patients, serum amyloid A showed a strong correlation with mucosal inflammation compared to C-reactive protein and had excellent sensitivity and specificity rates with significant statistical significance.

Conclusions: Serum amyloid A is a more useful marker compared to C-reactive protein in predicting mucosal inflammation in ulcerative colitis patients in clinical remission.

Keywords: Mucosal healing, Serum amyloid a, Ulcerative colitis, C-reactive protein

\section{Background}

Patients with ulcerative colitis (UC) typically manifest symptoms such as rectal bleeding, persistent bloody diarrhea, increased stool frequency, and abdominal pain $[1,2]$. Moreover, UC is a chronic inflammatory disorder of the

\footnotetext{
* Correspondence: r-hayashi@hiroshima-u.ac.jp

${ }^{2}$ Department of Endoscopy, Hiroshima University Hospital, 1-2-3 Kasumi, Minami-ku, Hiroshima 734-8551, Japan

Full list of author information is available at the end of the article
}

colorectum characterized by repeated clinical remission and relapse [3]. UC is generally thought to be caused by various factors, such as genetic factors, immune abnormality, and environmental factors including intestinal bacteria [1]. However, its cause has not been elucidated yet, and its fundamental therapy has not yet been established. The goal of UC treatment is to achieve clinical remission in patients; however, recently, treatment options, such as immunomodulators and biological agents, have been

C C The Author(s). 2020 Open Access This article is licensed under a Creative Commons Attribution 4.0 International License, which permits use, sharing, adaptation, distribution and reproduction in any medium or format, as long as you give appropriate credit to the original author(s) and the source, provide a link to the Creative Commons licence, and indicate if changes were made. The images or other third party material in this article are included in the article's Creative Commons licence, unless indicated otherwise in a credit line to the material. If material is not included in the article's Creative Commons licence and your intended use is not permitted by statutory regulation or exceeds the permitted use, you will need to obtain permission directly from the copyright holder. To view a copy of this licence, visit http://creativecommons.org/licenses/by/4.0/ The Creative Commons Public Domain Dedication waiver (http://creativecommons.org/publicdomain/zero/1.0/) applies to the data made available in this article, unless otherwise stated in a credit line to the data. 
gaining attention, and the therapeutic goal has changed from achieving clinical remission to achieving mucosal healing [1].

Mucosal healing is related to long-term clinical remission, and long-term prognosis is improved by reducing the risk of hospitalization and surgical operation [4]. Mucosal healing is detected via endoscopy, as colonoscopy is quite an invasive examination, and frequent examinations are difficult because of its medical cost. Therefore, in the diagnosis and treatment of UC, noninvasive, low-cost prediction methods of mucosal healing using useful biomarkers are clinically required. Generally, C-reactive protein (CRP) is reported to be less sensitive in UC cases [5], and it mildly increases in UC than in Crohn's disease (CD). Although serum amyloid A protein (SAA) is mainly secreted from the liver, similar to CRP $[6,7]$, SAA is reported to be more effective than CRP in diseases other than inflammatory bowel disease (IBD) [8]. In addition, SAA was reportedly correlated with the clinical activity in $\operatorname{UC}[9,10]$, but there is no report examining its correlation with endoscopic findings. Therefore, this study aimed to evaluate whether SAA is a better serum biomarker than CRP in predicting mucosal healing in UC patients in clinical remission.

\section{Methods \\ Patients}

This study included consecutive outpatients or inpatients who underwent endoscopic examinations at Hiroshima University from April 2010 to March 2017. CRP and SAA values of these patients measured within 1 month before and after colonoscopy were retrospectively analyzed. The exclusion criteria were as follows: patients who were administered new therapies during the period from colonoscopy to the time when CRP and SAA measurements were taken, those with other infections such as common cold, those with concurrent autoimmune diseases such as collagen diseases, and those who were not in clinical remission. UC diagnosis was made based on the clinical, endoscopic, and pathological findings. Demographic, clinical, endoscopic, and laboratory data were obtained from patients' medical records. Clinical symptoms were evaluated using the Rachmilewitz clinical activity index (CAI). Self-exclusive symptoms (weekly frequency of bowel movement, bloody stools, and abdominal pain), objective symptoms (temperature and investigator's assessment of symptomatic state), extraintestinal manifestations, and blood test findings (sedimentation rate and hemoglobin) were divided into seven items and evaluated using the total score (range: 0 to 29). Clinical remission was defined as a CAI of 4 or less [11]. In addition, the Montreal classification was used to define the extent of the lesion, which was classified into three types: ulcerative proctitis (E1), leftsided UC (E2), and extensive UC (E3) [12].

\section{Evaluation via endoscopic examination}

Mayo endoscopic subscore (MES) was used to evaluate the degree of mucosal inflammation in each part of the colorectum (cecum, ascending, transverse, descending, sigmoid colon, and rectum). Mucosal inflammation was analyzed using the maximum value among the scores. Mucosal healing was defined as MES 0 or 1 , whereas nonmucosal healing as MES 2 or 3 throughout the colorectum. Furthermore, complete mucosal healing (cMH) was defined as MES 0. The evaluation of inflammation was performed by three physicians with endoscopic experience of 7 years or more. A majority vote was adopted during disagreements of opinions. Moreover, when endoscopic score was judged, clinical symptoms were blinded.

\section{SAA and CRP measurements}

To measure SAA and CRP, blood specimens collected within 1 month before and after the colonoscopy were placed in a blood collection tube for biochemistry. SAA and CRP values were measured via an automatic analyzer using a latex agglutination reaction. The measurement kit for CRP was LZ Test Eiken CRP - HG and that for SAA was LZ Test Eiken SAA (Eikenkagaku, Tokyo, Japan). The measurement range was from 0.01 to $30 \mathrm{mg} / \mathrm{dL}$ for CRP and from 5 to $500 \mu \mathrm{g} / \mathrm{mL}$ for SAA. Both CRP and SAA used BM6070 (JEOL, Tokyo, Japan) as measuring equipment. In our hospital, when the SAA value was less than 5.6 , the examination value was displayed as " $<5.6$." If the test result was less than 5.6, the value was set to 5.5, and statistical analysis was performed.

\section{Statistical analysis}

All statistical analyzes were performed using EZR (Saitama Medical Center, Jichi Medical Center), a graphical user interface for $\mathrm{R}$ (The $\mathrm{R}$ Foundation for Statistical Computing, version 2.13.0) [13]. Spearman's rank correlation was used to analyze the correlation between the test result (SAA and CRP), MES, and endoscopic findings. MES and inspection values (SAA and CRP) were evaluated using the Cochran-Armitage trend test. A receiver's operating characteristic (ROC) curve was drawn to measure the area under the ROC curve and set a cutoff value. To infer the mucosal condition based on SAA and CRP values, sensitivity, specificity, positive predictive value (PPV), negative predictive value (NPV), and accuracy with $95 \%$ confidence interval were calculated. A $p$ value $<0.05$ was considered to indicate a statistically significant difference. 


\section{Ethical statement}

Our study protocol conformed to the ethical standards of the responsible committees on human experimentation (institutional and national) and with the Declaration of Helsinki in 1964 and later versions and was approved by the Institutional Review Board of Hiroshima University Hospital. In this study, we only used clinical information without invasion or intervention to the patient, and we disclosed information such as research implementation and purpose and secured participants' opportunity to refuse participation through posters.

\section{Results}

\section{Patient characteristics}

The patients' background is shown in Table 1. A total of 199 colonoscopies were performed in 108 UC patients who underwent blood tests for CRP and SAA (63 men, 45 women). The median age at the time of endoscopic examination was 44 (range, 12-72) years, and the median disease duration at endoscopic examination was 9 (0-38) years. The median CAI value was 0 (range, $0-4$ ) points. The Montreal classification was used to define the lesion range. The following number of cases were obtained: E1, 38 (19.1\%); E2, 47 (23.6\%); and E3, 114 (57.3\%). The median MES was 1 (range, 0-3). Even in clinical remission patients, $39.6 \%$ of patients had endoscopic activity. The median CRP was 0.04 (range, 0.021.09 ) $\mathrm{mg} / \mathrm{dL}$, and the median SAA was 5.5 (range, 5.590.1) $\mu \mathrm{g} / \mathrm{mL}$. For treatment, 89.4, 9.0, 35.7, and $12.1 \%$ of the patients were administered with mesalazine, corticosteroids, immunomodulators, and biologics, respectively.

\section{Correlation between SAA, CRP, and colon endoscopic findings}

We examined the correlation between SAA, CRP, and endoscopic findings. The results are shown in Fig. 1. A low correlation was found between CRP and MES $(r=0.352, p=$ $3.38 \times 10^{-7}$, Fig. 1a), whereas SAA had a high correlation with $\operatorname{MES}\left(r=0.614, p=5.44 \times 10^{-22}\right.$, Fig. $\left.1 \mathrm{~b}\right)$. At MES 0 , the ratio at which CRP and SAA are normal is the highest, and with increasing MES, the ratio gradually decreases. The decreasing trend in relation to the MES was statistically significant in SAA $(p<0.05)$, but not in CRP (Fig. 1a, b). The correlation between CRP, SAA, and MES was also examined in the same way, including in patients with no clinical remission $(\mathrm{CAI}>5)$. Both CRP and SAA were highly correlated with MES, and SAA was more strongly associated with MES. CRP showed a stronger correlation than in clinical remission patients alone (data not shown).

Table 1 Patient characteristics

\begin{tabular}{|c|c|}
\hline Total no. of patients & 108 \\
\hline Sex: male/female & $63 / 45$ \\
\hline Total colonoscopy & 199 \\
\hline \multicolumn{2}{|l|}{ Number of colonoscopies } \\
\hline 1 & 58 \\
\hline 2 & 30 \\
\hline$\geqq 3$ & 20 \\
\hline Age during colonoscopy: median (range) & $44(12-72)$ years \\
\hline Disease duration during colonoscopy: median (range) & $9(0-38)$ years \\
\hline \multicolumn{2}{|l|}{ Montreal classification (\%) } \\
\hline E1/E2/E3 & $38(19.1) / 47(23.6) / 114(57.3)$ \\
\hline \multicolumn{2}{|l|}{ Clinical score at colonoscopy: median (range) } \\
\hline CAl & $0(0-4)$ \\
\hline Mayo endoscopic score: median (range) & $1(0-3)$ \\
\hline \multicolumn{2}{|l|}{ Blood examination: median (range) } \\
\hline CRP & $0.04(0.02-1.09)$ \\
\hline SAA & $5.5(5.5-90.1)$ \\
\hline \multicolumn{2}{|l|}{ Therapies (\%) } \\
\hline Mesalamine & $178(89.4)$ \\
\hline Corticosteroids & $18(9.0)$ \\
\hline Immunomodulators & $71(35.7)$ \\
\hline Biologics & $24(12.1)$ \\
\hline
\end{tabular}

CAI Clinical activity index, CRP C-reactive protein, SAA Serum amyloid A 


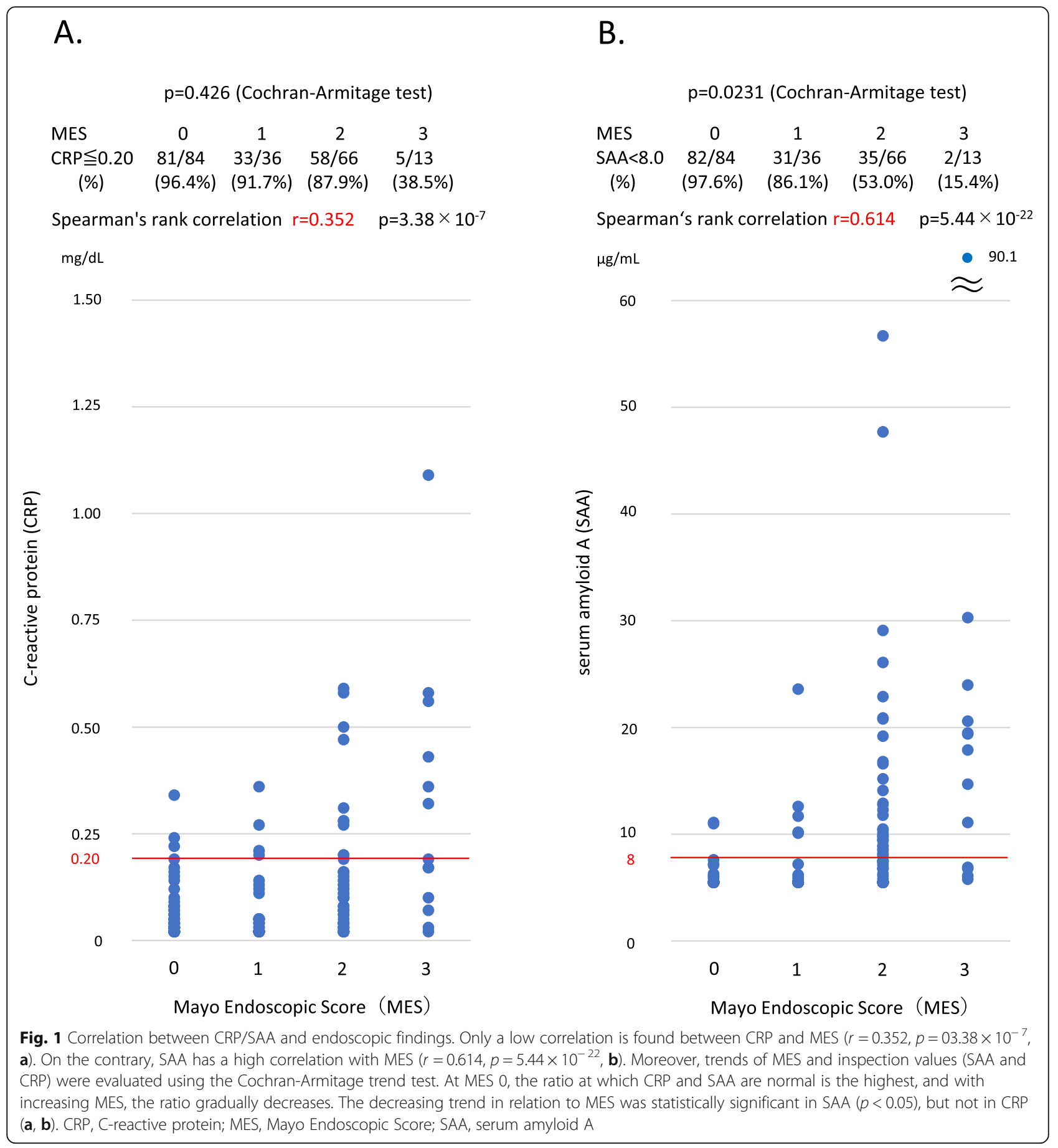

Diagnostic accuracy of SAA and CRP for mucosal healing Moreover, evaluation results of the predictive ability of SAA and CRP to identify mucosal inflammation in clinical remission patients are shown in Fig. 2. In the ROC analysis, the area under the ROC curve of the SAA was 0.807 , indicating a higher predictive power, whereas that of the CRP was 0.701. Comparison of ROC curves for mucosal inflammation of SAA and CRP showed that SAA was superior to CRP and indicated a statistical significance $(p<0.01)$. Table 2 shows the sensitivity, specificity, PPV, NPV, and accuracy of CRP and SAA at optimal cutoff values for mucosal inflammation in clinical remission patients. SAA levels $<5.8$ could discriminate mucosal inflammation from mucosal healing with sensitivity of 0.722 , specificity of 0.850 , PPV of 0.760 , NPV of 0.823 , and accuracy of 0.799 . On the contrary, CRP levels $<0.060$ could distinguish mucosal inflammation from mucosal healing with sensitivity of 0.620 , specificity 


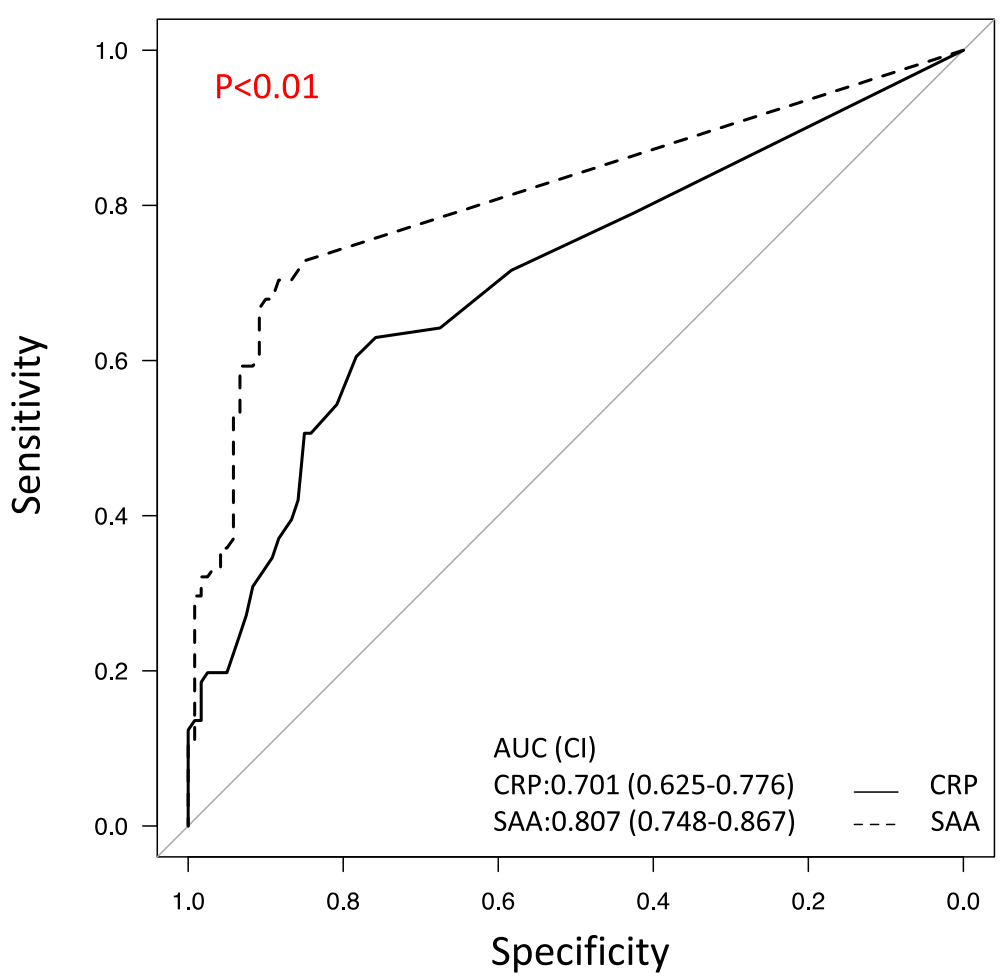

Fig. 2 Comparison of ROC curves for mucosal inflammation (MES 2 or 3) of SAA and CRP. The ROC curves of SAA and CRP in patients in clinical remission. In the ROC analysis, the area under the ROC curve of the SAA was 0.807 , indicating a higher predictive power, but that of CRP was 0.701. Comparison of ROC curves for mucosal inflammation (MES 2 or 3) of SAA and CRP showed that SAA was superior and indicated statistical significance $(p<0.01)$. AUC, area under the receiver's operating characteristic curve, $\mathrm{Cl}$, confidence interval; CRP, C-reactive protein; MES, Mayo Endoscopic Score; SAA, serum amyloid A

of 0.758 , PPV of 0.628 , NPV of 0.752 , and accuracy of 0.704 (Table 2). The results indicate that SAA could be an excellent marker in predicting mucosal healing in clinical remission patients than CRP. We compared the area under the ROC curve of SAA and CRP for mucosal inflammation, including patients who did not reach clinical remission $(\mathrm{CAI}>5)$. No significant difference was found (data not shown).

Recently, patients who achieved MES 0 have better prognosis compared to other patients [14, 15], and a higher treatment target is required for mucosal healing. Therefore, sensitivity and specificity, among others, were

Table 2 Ability to predict mucosal inflammation (MES 2 or 3) with optimal cutoff value by ROC curve

\begin{tabular}{lll}
\hline & CRP & SAA \\
\hline Sensitivity (95\% Cl) & $0.620(0.504-0.727)$ & $0.722(0.609-0.817)$ \\
Specificity (95\% Cl) & $0.758(0.672-0.832)$ & $0.850(0.773-0.909)$ \\
PPV (95\% Cl) & $0.628(0.511-0.735)$ & $0.760(0.647-0.851)$ \\
NPV (95\% Cl) & $0.752(0.665-0.826)$ & $0.823(0.744-0.885)$ \\
Accuracy (95\% Cl) & $0.704(0.635-0.766)$ & $0.799(0.737-0.852)$ \\
\hline
\end{tabular}

Cl Confidence interval, CRP C-reactive protein, MES Mayo Endoscopic Score, NPV Negative predictive interval, PPV Positive predictive value, SAA Serum amyloid $\mathrm{A}$ calculated by using mucosal healing only as MES 0 using the same method described above, and a ROC curve was drawn in clinical remission patients. The ROC, sensitivity, specificity, PPV, NPV, and accuracy with optimal cutoff values of SAA and CRP are shown in Fig. 3 and Table 3. Comparison of ROC curves for mucosal inflammation showed that SAA was superior than CRP, with statistically significant difference $(p<0.01)$, but its sensitivity was low.

In addition, we examined by disease duration about patients in clinical remission. Disease duration was divided into three groups: $0-5$ years $(N=69), 6-15$ years $(N=88)$, and more than 16 years $(N=42)$. We examined the ability of SAA and CRP to predict mucosal inflammation by comparing the areas under the ROC curve. There was no difference between the three groups regarding disease type, age, and CAI (data not shown). In the groups with disease duration of $0-5$ years and 6-15 years, SAA was significantly better than CRP, but in the group with disease duration of 16 years or more, the area under the ROC curve of CRP was high and SAA was not significant (Fig. 4).

We also examined disease type. Patients in clinical remission were divided into two groups: proctitis or left colitis $(\mathrm{E} 1+\mathrm{E} 2)$ and total colitis (E3). The area under the ROC curve of SAA and CRP for predicting mucosal 


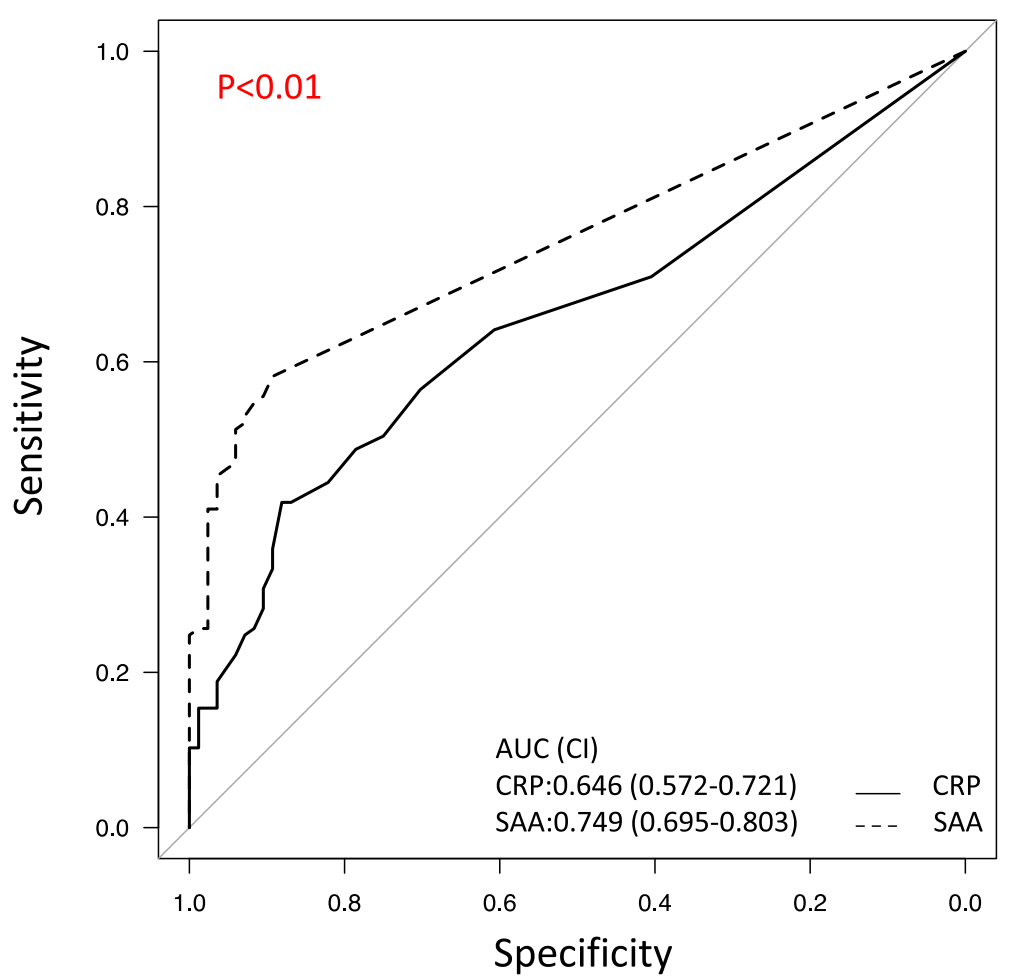

Fig. 3 Comparison of ROC curves for mucosal inflammation (MES 1 or 2 or 3) of SAA and CRP. The ROC curves of SAA and CRP in patients in clinical remission. In the ROC analysis, the area under the ROC curve of the SAA was 0.749 , indicating a higher predictive power, whereas that of the CRP was 0.646. Comparison of ROC curves for mucosal inflammation (MES 1 or 2 or 3) of SAA and CRP showed that SAA was superior and indicated statistical significance $(p<0.01)$. AUC, area under the receiver's operating characteristic curve; $\mathrm{Cl}$, confidence interval; $C R P, C$-reactive protein; MES, Mayo Endoscopic Score; SAA, serum amyloid A

inflammation in each group was compared. There was a significant difference in the E3 group, but there was no significant difference in the E1 + E2 group (data not shown). There was no difference between the two groups regarding age, disease duration, and CAI (data not shown). Therefore, the usefulness of SAA may be more enhanced in the group with widespread inflammation such as total colitis.

\section{Discussion}

In this study, we examined whether SAA better predicts mucosal healing in UC patients in clinical remission

Table 3 Ability to predict mucosal inflammation (MES 1 or 2 or 3) with optimal cutoff value by ROC curve

\begin{tabular}{lll}
\hline & CRP & SAA \\
\hline Sensitivity (95\% Cl) & $0.557(0.461-0.649)$ & $0.574(0.478-0.666)$ \\
Specificity (95\% Cl) & $0.702(0.593-0.797)$ & $0.893(0.806-0.950)$ \\
PPV (95\% Cl) & $0.719(0.614-0.809)$ & $0.880(0.784-0.944)$ \\
NPV (95\% Cl) & $0.536(0.439-0.632)$ & $0.605(0.513-0.691)$ \\
Accuracy (95\% Cl) & $0.618(0.547-0.686)$ & $0.709(0.640-0.771)$ \\
\hline
\end{tabular}

CI Confidence interval, CRP C-reactive protein, MES Mayo Endoscopic Score, NPV Negative predictive interval, PPV Positive predictive value, SAA Serum amyloid $\mathrm{A}$ compared to CRP. Our findings revealed that SAA has a strong correlation with endoscopic findings and is an excellent serum biomarker for predicting endoscopic activity in this patient cohort.

Monitoring of disease activity in routine practice is an important aspect in the clinical management of UC patients. It is very important to periodically examine clinical symptoms and endoscopic findings in such a population to determine the state of the colonic mucosa. However, frequent endoscopic examinations are difficult to perform; hence, biomarkers reflecting endoscopic findings are important.

Recently, fecal calprotectin has been used to evaluate mucosal inflammation, and its effectiveness has also been reported. Measuring fecal calprotectin levels has been proposed as a noninvasive test for evaluation of intestinal inflammation in IBD patients $[16,17]$. However, because of the complexity of collecting feces, the lack of the result on the same day in some hospitals, and fluctuating values even when measured on the same day, fecal calprotectin might not remain clinically useful [18].

Therefore, we considered that SAA used as an inflammatory marker might predict mucosal healing in UC. CRP and SAA are secreted mainly by hepatocytes produced in response to infection, trauma, and other 


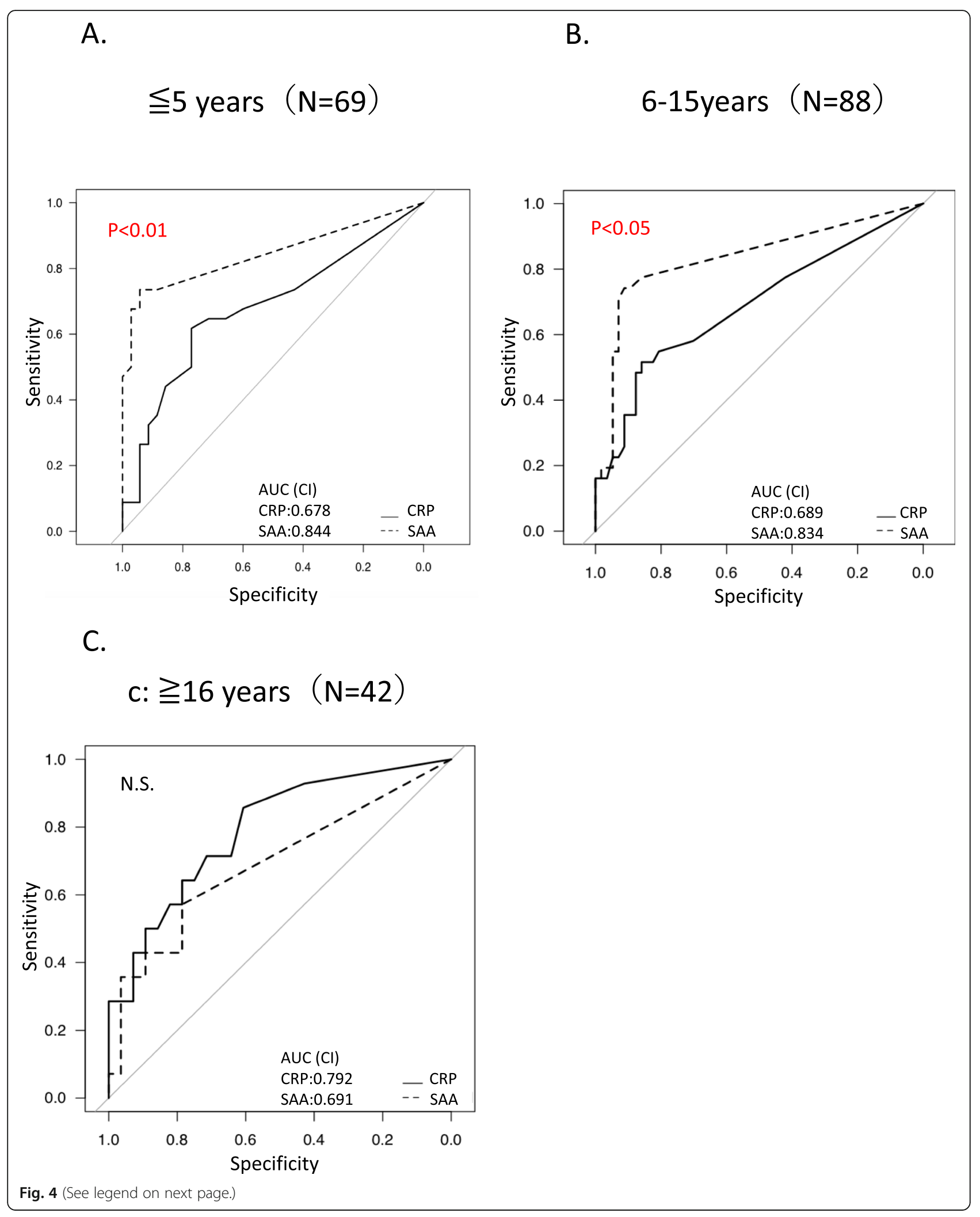


(See figure on previous page.)

Fig. 4 Comparison of ROC curves of mucosal inflammation (MES 2 or 3) of SAA and CRP when divided by disease duration. The ROC curves of SAA and CRP in patients in clinical remission when divided by disease duration. Disease duration was divided into three groups: $0-5$ years $(N=$ 69), 6-15 years $(N=88)$, and more than 16 years $(N=42)$. When examined by disease duration, comparison of ROC curves for mucosal inflammation (MES 2 or 3) of SAA and CRP showed that SAA was superior and indicated statistical significance in groups with disease duration of 0-5 years and 6-15 years. On the other hand, no statistical significance was shown in the group with disease duration of 16 years or more. AUC, area under the receiver's operating characteristic curve; CRP, C-reactive protein; MES, Mayo Endoscopic Score; SAA, serum amyloid A

inflammatory conditions [6, 7]. These serum concentrations increase sharply and slowly return to normal levels over several days. However, chronic inflammation causes a sustained increase of these serum concentrations [6, 7]. Although there is a positive correlation between CRP and SAA concentrations $[8,19]$, studies have shown that SAA can be a more sensitive marker of inflammation in certain diseases, such as rheumatoid arthritis, primary biliary cirrhosis, and chronic active hepatitis [8]. Therefore, we decided to evaluate whether SAA measurement is a better serum biomarker than CRP. A recently published study revealed that SAA correlates with endoscopic findings in patients with CD and that SAA can be a useful biomarker to predict mucosal healing [20]. On the other hand, there is no report examining the correlation between UC and endoscopic findings yet. Hence, this report is the first to describe the correlation between SAA and intestinal mucosal inflammation. In UC patients, there was a positive correlation between mucosal inflammation and SAA, with the correlation being stronger than that of CRP, and SAA was found to more accurately reflect the state of the mucosa. In comparison with CRP, SAA proved to be an excellent marker for predicting mucosal inflammation in clinical remission patients. Although the therapeutic goal of UC is mucosal healing, clinical and endoscopic findings do not necessarily match. Actually, even in this study, 39.6\% of clinical remission patients did not achieve mucosal healing. Therefore, among the clinical remission patients without symptoms, it is clinically important to evaluate intestinal inflammation using biomarkers than through frequent endoscopies. Although fecal markers, such as calprotectin, are considered useful, there are also limitations, as described above. Moreover, although CRP is still the most widely used serum biomarker, the existence of serum biomarkers that can more accurately predict mucosal healing is ideal.

Endoscopic examinations should be considered in clinical remission patients with elevated SAA, even if the CRP results are negative. Moreover, considering that measuring SAA is inexpensive and that the results are known on the same day, more facilities can adopt this approach and the financial burden on patients can be reduced. Excellent clinical outcome of patients with UC showing $\mathrm{CMH}$ (defined as MES 0) [15] has been set as the clinical goal in the treatment and management of these patients. SAA is an excellent marker for predicting mucosal inflammation than CRP. However, its diagnostic rate is lower when mucosal healing is defined as MES 0 or 1 , indicating that SAA is an excellent marker in predicting strong mucosal inflammation only to some extent. We also compared the area under the ROC curve of SAA and CRP to predict mucosal inflammation in all patients, including those who did not reach clinical remission $(\mathrm{CAI}<5)$. No significant difference was found. When the disease activity of UC increases, CRP level also tends to increase, and the significance of SAA decreases. Thus, SAA can be a better monitoring tool to predict mucosal inflammation than CRP in patients with clinical remission with low disease activity.

SAA is produced by the liver; it has recently been reported that it is also produced extrahepatically (Intestinal epithelium) [21]. In this study, SAA was not superior to CRP as a marker to predict mucosal inflammation when disease duration was prolonged. Longer disease duration may make it difficult for SAA to develop due to scarring and mucosal atrophy.

This study has several limitations. First, it is a retrospective study at a single facility involving a small absolute number of patients with UC. Excluding patients who were administered new therapies during the period from colonoscopy to the time CRP and SAA measurements were taken and those who were not in clinical remission may also be a selection bias. In addition, although no new treatment has been introduced in the included patients, the patients' condition may have slightly changed because the date of the endoscopic examination and the date of the blood test are different. Second, since not all patients underwent urine tests, chest X-ray examination, computed tomography, etc., we cannot completely exclude infectious diseases and malignant tumors that may have caused the elevated CRP and SAA levels. Further prospective studies that are able to address these problems are needed. Third, we did not compare SAA with fecal markers, such as calprotectin. Calprotectin, in spite of its limitations as we mentioned above, is the most well established marker of mucosal disease at present. A future study comparing the serum markers with fecal markers could be interesting. The strengths of the study were as follows: it demonstrated the correlation between endoscopic findings of UC and SAA, and because it is a blood test, which can be easily measured, we believe that it can be applied immediately in the clinical setting. 


\section{Conclusions}

In conclusion, SAA has a strong correlation with endoscopic findings and is an excellent marker than CRP for predicting endoscopic activity in UC patients in clinical remission.

\section{Abbreviations}

CAl: Clinical activity index; CD: Crohn's disease; cMH: complete mucosal healing; CRP: C-reactive protein; IBD: Inflammatory bowel disease; NPV: Negative predictive value; PPV: Positive predictive value; ROC: receiver's operating characteristic; SAA: Serum amyloid A protein; MES: Mayo endoscopic subscore

\section{Acknowledgements}

This work was not supported by any specific grant from any funding agency in the public, commercial, or not-for-sectors. We would like to thank Editage (www.editage.jp) for English language editing.

\section{Availability of data or materials}

The datasets used and analysed during the current study are available from the corresponding author on reasonable request.

\section{Authors' contributions}

All authors have read and approved the manuscript in its latest state. K.C. S.T., Y.U., R.H., and M.W. designed this study. M.W., T.N., J.K., M.N., H. T, and S.O. performed colonoscopy and assessed endoscopic findings. R.H., Y.U., S.T., S.O., and M.I. treated patients and assessed clinical findings. M.W. wrote this manuscript and performed the statistical analysis with contributions from R.H., Y.U., S.T., M.I., and K.C.

\section{Funding}

Not applicable.

\section{Ethics approval and consent to participate}

Our study protocol conformed to the ethical standards of the responsible committees on human experimentation (institutional and national) and with the Declaration of Helsinki in 1964 and later versions and was approved by the Institutional Review Board of Hiroshima University Hospital. In this study, we only used clinical information without invasion or intervention to the patient, and we disclosed information such as research implementation and purpose and secured participants' opportunity to refuse participation through posters.

\section{Consent for publication}

Not applicable.

\section{Competing interests}

The authors declare that they have no competing interests.

\section{Author details}

'Department of Gastroenterology and Metabolism, Hiroshima University Hospital, 1-2-3 Kasumi, Minami-ku, Hiroshima 734-8551, Japan. ${ }^{2}$ Department of Endoscopy, Hiroshima University Hospital, 1-2-3 Kasumi, Minami-ku, Hiroshima 734-8551, Japan.

Received: 13 March 2019 Accepted: 20 March 2020

Published online: 03 April 2020

\section{References}

1. Danese S, Fiocchi C. Ulcerative colitis. N Engl J Med. 2011:365:1713-25.

2. Colombel JF, Rutgeerts P, Reinisch W, et al. Early mucosal healing with infliximab is associated with improved long-term clinical outcomes in ulcerative colitis. Gastroenterology. 2011;141:1194-201.

3. Langholz E, Munkholm P, Davidsen M, Binder V. Course of ulcerative colitis: analysis of changes in disease activity over years. Gastroenterology. 1994;07:3-11.

4. Pineton de Chamburn G, Peyrin-Biroulet L, Lemann M, Colombel JF. Clinical implications of mucosal healing for the management of IBD. Nat Rev Gastroenterol Hepatol. 2010;7:15-29.

5. Fagan EA, Dyck RF, Maton PN, et al. Serum level of C-reactive protein in Crohn's disease and ulcerative colitis. Eur J Clin Investig. 1982;12:351-9.
6. Gabay C, Kushner I. Acute-phase proteins and other systemic responses to inflammation. N Engl J Med. 1999:340:448-54.

7. MacGregor AJ, Gallimore JR, Spector TD, Pepys MB. Genetic effects on baseline values of $\mathrm{C}$-reactive protein and serum amyloid a protein: a comparison of monozygotic and dizygotic twins. Clin Chem. 2004;50:130-4.

8. Raynes JG, Cooper EH. Comparison of serum amyloid a protein and Creactive protein concentrations in cancer and non-malignant disease. J Clin Pathol. 1983;36:798-803.

9. Chambers RE, Stross P, Barry RE, Whicher JT. Serum amyloid a protein compared with C-reactive protein, alpha 1-antichymotrypsin and alpha 1acid glycoprotein as a monitor of inflammatory bowel disease. Eur J Clin Investig. 1987;17:460-7.

10. Niederau C, Backmerhoff F, Schumacher B, Niederau C. Inflammatory mediators and acute phase proteins in patients with Crohn's disease and ulcerative colitis. Hepatogastroenterology. 1997:44:90-107.

11. Rachmilewitz D. Coated mesalazine (5-aminosalicylic acid) versus sulphasalazine in the treatment of active ulcerative colitis: a randomized trial. BMJ. 1989;298:82-6.

12. Satsangi J, Silverberg MS, Vermeire S, Colombel JF. The Montreal classification of inflammatory bowel disease: controversies, consensus, and implications. Gut. 2006;55:749-53.

13. Kanda Y. Investigation of the freely available easy-to-use software 'EZR' for medical statistics. Bone Marrow Transplant. 2013;48:452-8.

14. Manginot C, Baumann C, Peyrin-Biroulet L. An endoscopic Mayo of 0 is associated with a lower risk of colectomy than a score of 1 in ulcerative colitis. Gut. 2015;64:1181-2.

15. Barreiro-de Acosta M, Vallejo N, de la Iglesia D, Uribarri L, Bastón I, FerreiroIglesias $\mathrm{R}$, et al. Evaluation of the risk of relapse in ulcerative colitis according to the degree of mucosal healing (Mayo 0 vs 1): a longitudinal cohort study. J Crohn Colitis. 2016;10:13-9.

16. Costa F, Mumolo MG, Bellini M, et al. Role of faecal calprotectin as a noninvasive marker of intestinal inflammation. Dig Liver Dis. 2003;35:642-7.

17. Limburg PJ, Ahlquist DA, Sandborn WJ, et al. Faecal calprotectin levels predict colorectal inflammation among patients with chronic diarrhea referred for colonoscopy. Am J Gastroenterol. 2000:95:2831-7.

18. Lasson A, Stotzer PO, Öhman O, et al. The intra-individual variability of faecal calprotectin: a prospective study in patients with active ulcerative colitis. J Crohns Colitis. 2015;9:26-32.

19. Maury CP. Comparative study of serum amyloid a protein and C-reactive protein in disease. Clin Sci. 1985;68:233-8.

20. Ishihara S, Tada Y, Kawashima K, Kataoka M, Sonoyama H, Yamashita N, et al. Serum amyloid a level correlated with endoscopic findings in patients with Crohn's disease-possible biomarker for evaluating mucosal healing. Dig Liver Dis. 2018:50:553-8.

21. Erik RM. Eckhardt, Jassir Witta, Jian Zhong et al. intestinal epithelial serum amyloid a modulates bacterial growth in vitro and pro-inflammatory responses in mouse experimental colitis. BMC Gastroenterol. 2010;10:133.

\section{Publisher's Note}

Springer Nature remains neutral with regard to jurisdictional claims in published maps and institutional affiliations.

Ready to submit your research? Choose BMC and benefit from:

- fast, convenient online submission

- thorough peer review by experienced researchers in your field

- rapid publication on acceptance

- support for research data, including large and complex data types

- gold Open Access which fosters wider collaboration and increased citations

- maximum visibility for your research: over $100 \mathrm{M}$ website views per year

At BMC, research is always in progress.

Learn more biomedcentral.com/submission 